\title{
A Morbidity Survey of South African Primary Care
}

\author{
Bob Mash ${ }^{1 *}$, Lara Fairall ${ }^{2}$, Olubunmi Adejayan ${ }^{2}$, Omozuanvbo Ikpefan $^{2}$, Jyoti Kumari ${ }^{2}$, Shaheed \\ Mathee $^{2}$, Ronit Okun², Willy Yogolelo²
}

1 Division of Family Medicine and Primary Care, Stellenbosch University, Cape Town, Western Cape, South Africa, 2 Knowledge Translation Unit, Lung Institute, University of Cape Town, Cape Town, Western Cape, South Africa

\begin{abstract}
Background: Recent studies have described the burden of disease in South Africa. However these studies do not tell us which of these conditions commonly present to primary care providers, how these conditions may present and how providers make sense of them in terms of their diagnoses. Clinical nurse practitioners are the main primary care providers and need to be better prepared for this role. This study aimed to determine the range and prevalence of reasons for encounter and diagnoses found among ambulatory patients attending public sector primary care facilities in South Africa.

Methodology/Principal Findings: The study was a multi-centre prospective cross-sectional survey of consultations in primary care in four provinces of South Africa: Western Cape, Limpopo, Northern Cape and North West. Consultations were coded prior to analysis by using the International Classification of Primary Care-Version 2 in terms of reasons for encounter (REF) and diagnoses. Altogether 18856 consultations were included in the survey and generated 31451 reasons for encounter (RFE) and 24561 diagnoses. Women accounted for 12526 (66.6\%) and men 6288 (33.4\%). Nurses saw 16238 $(86.1 \%)$ and doctors $2612(13.9 \%)$ of patients. The top 80 RFE and top 25 diagnoses are reported and ongoing care for hypertension was the commonest RFE and diagnosis. The 20 commonest RFE and diagnoses by age group are also reported.
\end{abstract}

Conclusions/Significance: Ambulatory primary care is dominated by non-communicable chronic diseases. HIV/AIDS and TB are common, but not to the extent predicted by the burden of disease. Pneumonia and gastroenteritis are commonly seen especially in children. Women's health issues such as family planning and pregnancy related visits are also common. Injuries are not as common as expected from the burden of disease. Primary care providers did not recognise mental health problems. The results should guide the future training and assessment of primary care providers.

Citation: Mash B, Fairall L, Adejayan O, Ikpefan O, Kumari J, et al. (2012) A Morbidity Survey of South African Primary Care. PLoS ONE 7(3): e32358. doi:10.1371/ journal.pone.0032358

Editor: John E. Mendelson, California Pacific Medical Center Research Institute, United States of America

Received November 4, 2011; Accepted January 20, 2012; Published March 16, 2012

Copyright: (c) 2012 Mash et al. This is an open-access article distributed under the terms of the Creative Commons Attribution License, which permits unrestricted use, distribution, and reproduction in any medium, provided the original author and source are credited.

Funding: This study was supported by funds from the National Research Foundation (http://nrfonline.nrf.ac.za/) of South Africa's Incentive Scheme for Rated Researchers (Prof Bob Mash) and by funds held by PALSA Plus, Lung Institute, University of Cape Town. The funders had no role in study design, data collection and analysis, decision to publish, or preparation of the manuscript.

Competing Interests: The authors have declared that no competing interests exist.

*E-mail: rm@sun.ac.za

\section{Introduction}

After the fall of Apartheid in 1994 the new government of South Africa committed itself to a district health system based on the principles of primary health care. Implementation of this policy involved the integration of fragmented health departments and the rapid expansion of access to care through building more clinics, making services free and placing the nurse practitioner on the front line. Nurses were supported by medical officers and more recently a new cadre of specialist family physicians has been introduced with responsibility for clinical governance, consulting more complex patients as well as mentoring and support of primary care providers.

The 2008 World Health Report Primary Health Care - Now More Than Ever reinforced the need for countries to implement primary health care. [1] In 2011 the South African government recognized that, while much has been achieved in terms of infrastructure and access to care, the country is still not getting value for money through its primary health care system. [2] For example South Africa is one of the few countries where infant mortality rates have been increasing despite the Millennium Development Goals and spending $8.3 \%$ of the GDP on health. [3] In light of this there is currently an active debate on the re-engineering of primary health care and an interest in the lessons that can be learnt from the Brazilian model and family health care teams. [4] If South Africa adopted such a model then primary health care teams would most likely consist of community health workers, professional nurses, clinical nurse practitioners and supporting medical officers or family physicians.

The South African burden of disease study has used disability adjusted life years (DALYs) to estimate the contribution of different diseases to mortality and morbidity at a community level. [5] The study supports the concept of a quadruple burden of disease with the largest burden derived from HIV/AIDS and TB. The other quadrants include interpersonal violence and trauma, maternal and child health issues and non-communicable chronic diseases. The burden of disease study has been invaluable in aligning health system planning and academic curricula with the needs of the country. However it does not tell us which of these conditions commonly present to primary care providers, how these 
Table 1. Summary of sampling strategy.

\begin{tabular}{|c|c|c|c|c|c|c|}
\hline \multirow[t]{2}{*}{ Sub-district } & \multirow[t]{2}{*}{ Location } & \multirow[t]{2}{*}{ Population } & \multirow[t]{2}{*}{ Health workers } & \multicolumn{3}{|c|}{ Required number of facilities } \\
\hline & & & & CHC & Clinic & Mobile \\
\hline \multicolumn{7}{|c|}{ WESTERN CAPE PROVINCE } \\
\hline Klipfontein & Urban & 341,489 & 17 & 2 & 4 & 0 \\
\hline Tygerberg & Urban & 434,896 & 22 & 2 & 5 & 0 \\
\hline Saldanha Bay & Rural & 78,825 & 11 & 1 & 3 & 2 \\
\hline Swartland & Rural & 76,436 & 10 & 1 & 1 & 4 \\
\hline \multicolumn{7}{|l|}{ LIMPOPO PROVINCE } \\
\hline Ba-Phalaborwa & Rural & 143,410 & 12 & 1 & 3 & 1 \\
\hline Greater Letaba & Urban & 232,119 & 15 & 0 & 7 & 1 \\
\hline Greater Tzaneen & Urban & 393,867 & 26 & 1 & 8 & 3 \\
\hline Maruleng & Rural & 98,565 & 7 & 0 & 3 & 1 \\
\hline \multicolumn{7}{|l|}{ NORTHERN CAPE } \\
\hline Sol Plaajtie & Urban & 200,013 & 25 & 0 & 12 & 0 \\
\hline Dikgatlong & Rural & 39,881 & 17 & 0 & 9 & 0 \\
\hline Magareng & Rural & 21,348 & 6 & 0 & 3 & 0 \\
\hline Phokwane & Rural & 40,757 & 12 & 0 & 6 & 0 \\
\hline \multicolumn{7}{|l|}{ NORTH WEST } \\
\hline Ditsobotla & Rural & 157,922 & 13 & 1 & 4 & 2 \\
\hline Ramotshere Moiloa & Rural & 142,417 & 14 & 1 & 4 & 1 \\
\hline Ratlou & Rural & 108,317 & 11 & 1 & 3 & 1 \\
\hline Mafikeng & Urban & 270,008 & 22 & 1 & 8 & 1 \\
\hline
\end{tabular}

*Community Health Centre.

doi:10.1371/journal.pone.0032358.t001

conditions present or how providers make sense of them in terms of their diagnoses.

As primary care is the first point of contact with the health services it could be expected that the conditions seen would be correlated with the burden of disease (as measured by DALYs). Where a different pattern is noted this maybe because these conditions do not present to the health services, present in vertical programmes or other levels of the health system and not primary care, or are poorly recognized when they do present.

Understanding the nature of presentations in primary care will greatly assist with the training of primary care providers and ensure that they are competent to assess the common undifferentiated symptoms. It will also enable the development of tools and educational resources.

Mismatches between the expected burden of disease and the actual presentations and diagnoses may also enable critical reflection on whether primary care is effectively engaging with the burden of disease and how the system should be modified for a better fit.

Previous studies that address these issues in South Africa have been on a small scale, focused on a single practice or health centre, or are outdated in terms of the current burden of disease. $[6,7,8,9]$

This study aimed to determine the range and prevalence of reasons for encounter and diagnoses found among ambulatory patients attending public sector primary care facilities in South Africa.

\section{Methods}

\section{Ethics statement}

Ethical approval for the study was obtained from the Health Research Ethics Committees of the Universities of Stellenbosch and Cape Town and permission to conduct the study from the respective Provincial Departments of Health. Informed written consent was obtained from all health workers who participated in the study as approved by the ethics committees. Written consent was not required from the patients as no identifiers or additional information beyond that obtained in the usual consultation was collected and this was approved by the ethics committees.

\section{Study design}

The study was a multi-centre prospective cross-sectional survey of consultations in primary care in four provinces of South Africa: Western Cape, Limpopo, Northern Cape and North West. These provinces were purposefully selected since research assistants, registered as postgraduate students for a Masters in family medicine, were available for fieldwork in the regions.

\section{Setting}

In the South African setting about $16 \%$ of the population has insurance and makes use of the private sector. The remaining $84 \%$ of the population is dependent on the public sector, although some will pay cash for ad hoc use of the private sector.[10] The public sector primary care services are nurse-led with support from doctors. Primary care makes use of mobile clinics in remote areas to visit rural communities as well as fixed clinics. Clinics are usually only staffed by nurses and are themselves supported by larger community health centres. Community health centres are usually located in towns and urban areas and provide a wider range of health professionals and services, such as doctors, pharmacists, radiographers, or physiotherapists. Parts of the health centre may be dedicated to particular programmes or services such as HIV, TB or emergencies. 
The provinces selected represent $32 \%$ of the South African population and range in size from the Northern Cape $(1.8 \%)$ and North West $(8.2 \%)$ to the Western Cape $(10.2 \%)$ and Limpopo (11.7\%). Professional nurses range from 89 per 100,000 population in North West to 119 per 100,000 in Limpopo; and for medical practitioners from 11 per 100,000 in North West to 32 per 100,000 in the Western Cape. The percentage of rural communities also ranges from $11 \%$ in the Western Cape to $89 \%$ in Limpopo.[11] These provinces, therefore, included large metropolitan areas as well as rural towns and remote farming communities. They included different climatic zones and some malarious areas.

\section{Sampling}

The sample size was based on two considerations: firstly the number of health care workers a research assistant could train and support across a number of facilities and secondly on ensuring that the secondary reasons for encounter would be encountered in large enough numbers. The sample size per province was therefore the product of the number of health care workers that could be handled (60), the number of sampling days for each health care worker (5) and the number of patients per day (20) resulting in 6000 encounters per province and 24000 overall.

One district was purposefully selected from each Province based on the location of the research assistants. Out of these districts 4 sub-districts were purposively selected and at least 1 of the subdistricts was an urban area. Urban sub-districts were defined as having a town or metropolitan area and a population of more than 200,000 people. In the Western Cape sub-districts were selected from the Metropolitan and West Coast districts to enable a mix of rural and urban populations.

The sample size required from each sub-district to make up the total of 6000 for the Province was stratified according to the population of the sub-district. The facilities in each sub-district were then listed and divided into community health centres, fixed clinics or mobile clinics. It was assumed that a larger community health centre would have 5 health workers participating in the survey, a fixed clinic 2 health workers and a mobile clinic 1 health worker. It was also assumed that each health worker would see at least 20 patients a day and collect data on 5 separate days. The number of health workers required to deliver the sample size was then determined and distributed between the different types of facilities in proportion to the total number of different facilities in the sub-district (see Table 1). The required number of health centres, fixed clinics and mobile clinics were then randomly selected. In Tygerberg and Klipfontein the City of Cape Town, which runs the clinics, refused permission for the survey and therefore four community health centres were selected.

At each selected facility, health workers collected data on 5 separate days over a 1 year period. The first day was randomly chosen in the February-March period and then subsequent days booked every 2 months. Each of the 5 data collection days were also selected to be on a different day of the week so that each working day was covered once. This sampling strategy allowed for seasonal and daily variation in the patient presentations and diagnoses.

\section{Data collection}

At each selected facility the research assistant explained the project and invited primary care providers, either doctors or nurses, to participate. Health workers received a small shopping voucher after each data collection day to thank them for their time and commitment. Health workers were provided with a data collection tool which allowed them to record the age and sex of each patient and up to 5 reasons for encounter and 5 diagnoses for that consultation. No distinction was made between primary and secondary or ongoing diagnoses. Data were collected on all sequential ambulatory patients seen by the health worker on that day. Health workers were expected to be working in general primary care and not a specialised vertical programme or emergency department.

\section{Data analysis}

The International Classification of Primary Care Second Edition (ICPC-2) was used to code all reasons for encounter and diagnoses. [12] The ICPC-2 was developed by the World Organisation of National Colleges, Academies and Academic Associations of General Practitioners/Family Physicians (WONCA) as a classification system uniquely suited to primary care. The system enables classification of the reasons for encounter and diagnoses using a biaxial structure. The first axis codes the body system involved by means of a letter derived from 17 possible chapters (Table 2). The second axis contains 7 components related to different aspects of the consultation as shown in Table 2. Within each component a menu of standardised rubrics are listed with definitions, inclusion and exclusion criteria. These rubrics provide a two-digit numeric code that is combined with the letter to give the final classification. For example HIV/AIDS is coded as B90, type 2 diabetes as T90, tuberculosis as A70.

Table 2. ICPC-2 bi-axial classification system.

\begin{tabular}{ll}
\hline Axis & 1: Chapters based on body systems \\
\hline A & General and unspecified \\
B & $\begin{array}{l}\text { Blood, blood forming organs and immune mechanism (spleen, bone } \\
\text { marrow) }\end{array}$ \\
D & Digestive \\
F & Eye \\
H & Ear (Hearing) \\
K & Circulatory \\
L & Musculoskeletal (locomotion) \\
N & Neurological \\
P & Psychological \\
R & Respiratory \\
S & Skin \\
T & Endocrine, metabolic and nutritional \\
U & Urological \\
W & Pregnancy, child bearing, family planning \\
X & Female genital \\
Y & Male genital \\
Z & Social problems \\
\hline Axis & $2:$ Components of the consultation \\
\hline
\end{tabular}

Axis 2: Components of the consultation

\begin{tabular}{ll}
\hline 1 & Complaints and symptoms \\
2 & Diagnostic, screening and preventive activities \\
3 & Medication, treatment and procedures \\
4 & Test results \\
5 & Administrative activities \\
6 & Referrals and other reasons for encounter \\
7 & Diagnosis/diseases (infectious, neoplasms, injuries, congenital, other) \\
\hline
\end{tabular}




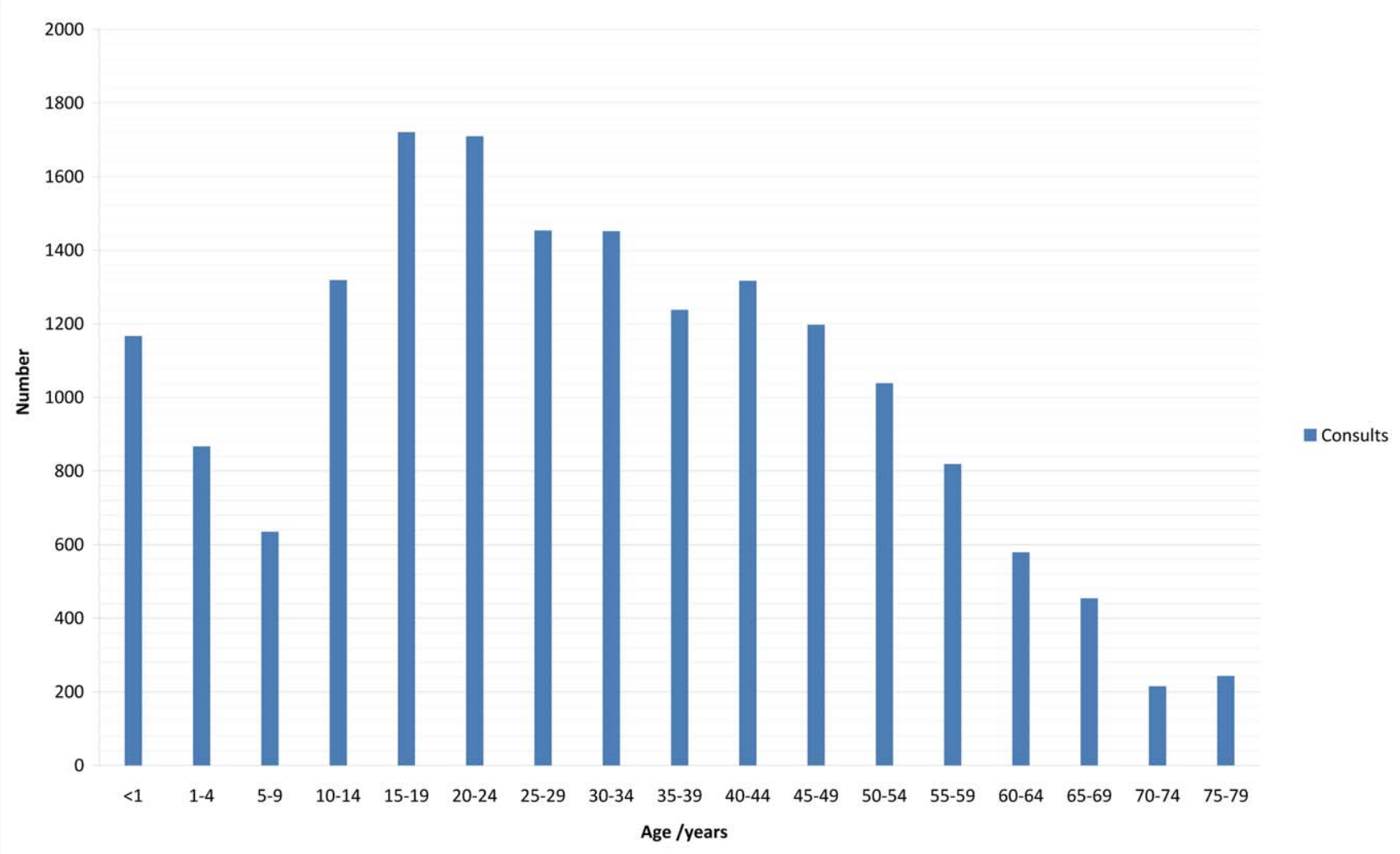

Figure 1. Distribution of number of consultations by age groups. doi:10.1371/journal.pone.0032358.g001

The research assistants in each Province were trained to code the data using ICPC-2 and each provided an excel sheet with the consultations already captured and coded. The combined data

Table 3. Distribution of RFEs between body systems in ICPC$2(\mathrm{~N}=31451)$.

\begin{tabular}{lll}
\hline & & \\
\hline ICPC-2 chapters & n \\
\hline Respiratory & 5499 & 17.5 \\
General and unspecified & 4521 & 14.4 \\
\hline Cardiovascular & 3327 & 10.6 \\
Digestive & 2839 & 9.0 \\
Musculoskeletal & 2685 & 8.5 \\
Pregnancy, child bearing, family planning & 2489 & 7.9 \\
Neurological & 2303 & 7.3 \\
Skin & 1646 & 5.2 \\
Endocrine, metabolic, nutritional & 1565 & 5.0 \\
Female genital & 1108 & 3.5 \\
\hline Blood, blood forming organs and immune system & 949 & 3.0 \\
Urological & 656 & 2.1 \\
\hline Eye & 625 & 2.0 \\
\hline Ear & 499 & 1.6 \\
Psychological & 398 & 1.3 \\
Male genital & 225 & 0.7 \\
Social problem & 117 & 0.4 \\
\hline doi:10.1371/journal.pone.0032358.t003 & & \\
\hline
\end{tabular}

were then analysed by the Centre for Statistical Consultation at Stellenbosch University. Descriptive statistics using frequency and means were calculated for the total data set, age groups, gender and provider type. The mean number of RFE and diagnoses for gender and provider type were compared by the Mann-Whitney U Test.

An error rate for each research assistant was analysed based on a representative random sample of their data sheets. The data sheets were coded independently by the principal researcher. The error rate for coding reasons for encounter was $11.3 \%$ (95\%CI 7.4-15.2) and for diagnoses was $11.1 \%$ (95\%CI 7.0-15.1).

Table 4. RFE associated with chronic care $(\mathrm{N}=31451)$.

\begin{tabular}{llc}
\hline Reason for encounter & $\mathbf{n}$ & $\%$ \\
\hline Cardiovascular e.g. hypertension (K31, K50, K61, K63, K64, K85) & 2976 & 9.5 \\
Women's health e.g. family planning, pregnancy & & \\
(W14, W31, W50, W64) & 2102 & 6.7 \\
Immunisations (A44) & 871 & 2.8 \\
Unspecified e.g. TB (A50, A64) & 892 & 2.8 \\
Immune e.g. HIV (B34, B50, B60) & 687 & 2.2 \\
Metabolic e.g. diabetes (T50, T64) & 618 & 2.0 \\
Neurological e.g. epilepsy (N50) & 248 & 0.8 \\
Respiratory e.g. asthma (R50) & 241 & 0.8 \\
Psychological e.g. schizophrenia (P50) & 183 & 0.6 \\
\hline Musculoskeletal e.g. arthritis (L50) & 78 & 0.2 \\
\hline doi:10.1371/journal.pone.0032358.t004 & &
\end{tabular}


Table 5. Commonest complaints in primary care $(\mathrm{N}=31451)$.

\begin{tabular}{|c|c|c|}
\hline Reason for encounter & $\mathbf{n}$ & $\%$ \\
\hline 1. Cough (R05) & 2821 & 9.0 \\
\hline 2. Headache (N01) & 1500 & 4.8 \\
\hline 3. Fever (A03) & 869 & 2.8 \\
\hline 4. Sneezing/nasal complaint (R07, R08) & 624 & 2.0 \\
\hline 5. Sore throat (R21) & 623 & 2.0 \\
\hline 6. Back pain (L02, L03) & 589 & 1.9 \\
\hline 7. Generalised aches or pains (A01) & 585 & 1.9 \\
\hline 8. Diarrhoea (D11) & 575 & 1.8 \\
\hline 9. Abdominal pain or cramp (D01) & 528 & 1.7 \\
\hline 10. Dysuria (U01) & 431 & 1.4 \\
\hline 11. Loss of appetite (T03) & 419 & 1.3 \\
\hline 12. Vomiting (D10) & 413 & 1.3 \\
\hline 13. Leg or thigh pain or cramps (L14) & 366 & 1.2 \\
\hline 14. Generalised rash (S07) & 318 & 1.0 \\
\hline 15. Vaginal discharge (X14) & 306 & 1.0 \\
\hline 16. Vertigo/dizziness (N17) & 299 & 0.9 \\
\hline 17. Localised rash (S06) & 290 & 0.9 \\
\hline 18. Ear pain $(\mathrm{H} 01)$ & 281 & 0.9 \\
\hline 19. Weakness/general tiredness (A04) & 277 & 0.9 \\
\hline 20. Pruritus (S02) & 247 & 0.8 \\
\hline 21. Abdominal pain, localised (D06) & 232 & 0.7 \\
\hline 22. Respiratory/pleuritic pain (R01) & 223 & 0.7 \\
\hline 23. Joint pain or symptoms (L20) & 212 & 0.7 \\
\hline 24. Knee pain or symptom (L15) & 190 & 0.6 \\
\hline 25. Shoulder pain or symptom (L08) & 187 & 0.6 \\
\hline 26. Shortness of breath (R02) & 180 & 0.6 \\
\hline 27. Chest pain (A11) & 178 & 0.6 \\
\hline 28. Foot and toe pain or symptoms (L17) & 165 & 0.5 \\
\hline 29. Weight loss (T08) & 165 & 0.5 \\
\hline 30. Swallowing problem (D21) & 156 & 0.5 \\
\hline 31. Hand and finger pain or symptom (L12) & 154 & 0.5 \\
\hline 32. Eye pain (F01) & 151 & 0.5 \\
\hline 33. Epigastric pain (D02) & 134 & 0.4 \\
\hline 34. Neck pain (L01) & 132 & 0.4 \\
\hline 35. Mouth, tongue, lip complaints (D20) & 128 & 0.4 \\
\hline 36. Eye discharge (F03) & 126 & 0.4 \\
\hline 37. Arm pain or symptom (L09) & 121 & 0.4 \\
\hline 38. Nausea (D09) & 120 & 0.4 \\
\hline 39. Menstruation absent/scanty (X05) & 118 & 0.4 \\
\hline 40. Sweating (A09) & 116 & 0.4 \\
\hline 41. Localized lump(s) or swelling(s) (S04) & 116 & 0.4 \\
\hline 42. Abnormal sputum (R25) & 114 & 0.4 \\
\hline 43. Respiratory complaint e.g. tight chest (R29) & 110 & 0.3 \\
\hline 44. Breathing problem (R04) & 108 & 0.3 \\
\hline 45. Genital/pelvic pain (X01) & 107 & 0.3 \\
\hline 46. Constipation (D12) & 106 & 0.3 \\
\hline 47. Ear discharge $(\mathrm{H} 04)$ & 100 & 0.3 \\
\hline 48. Skin complaint (S29) & 99 & 0.3 \\
\hline 49. Red eye (F02) & 96 & 0.3 \\
\hline 50. Teeth or gum complaint (D19) & 95 & 0.3 \\
\hline
\end{tabular}

Table 5. Cont.

\begin{tabular}{lll}
\hline & & \\
\hline Reason for encounter & $\mathbf{n}$ & $\%$ \\
\hline 51. Chest pain, musculoskeletal (L04) & 95 & 0.3 \\
52. Trauma/injury (A80) & 92 & 0.3 \\
53. Vaginal symptoms (X15) & 90 & 0.3 \\
54. Eye sensation, abnormal (F13) & 83 & 0.3 \\
55. Heartburn (D03) & 79 & 0.3 \\
56. Urethral discharge (Y03) & 78 & 0.2 \\
\hline
\end{tabular}

doi:10.1371/journal.pone.0032358.t005

\section{Results}

Altogether 18856 consultations were included in the survey and generated 31451 reasons for encounter (RFE) and 24561 diagnoses. Limpopo provided 6678 (35.4\%), Northern Cape $1504(7.9 \%)$, North-West 5082 (26.9\%) and Western Cape 5592 $(29.6 \%)$ of the consultations. Women accounted for $12526(66.6 \%)$ and men $6288(33.4 \%)$ of consultations. Women presented with a mean of $1.65 \mathrm{RFE}$ and men with significantly more at 1.69 $(\mathrm{p}<0.01)$. Primary care providers made a mean of 1.30 diagnoses in women and men.

Nurses saw 16238 (86.1\%) and doctors 2612 (13.9\%) of patients. Nurses had a mean of 1.65 reasons for encounter per consultation while doctors saw significantly more at a mean of $1.76(\mathrm{p}<0.05)$. Nurses made a mean of 1.24 diagnoses per encounter while doctors made significantly more at a mean of $1.69(\mathrm{p}<0.05)$.

The distribution of the consultations with age is shown in Figure 1. The distribution shows two peaks, amongst infants and in the late teens/young adult age categories.

How the reasons for encounter were distributed between the different bodily systems in ICPC-2 are shown in Table 3. Patients in primary care mostly presented with respiratory, unspecified and cardiovascular problems. Although psychosocial problems are undoubtedly common amongst the population using the public sector, these were not commonly stated as the RFE. Neurological conditions were relatively common, but 1500 of these counts were due to headache alone.

Table 4 and Table 5 combined represent the 80 most common RFE and together also make up 26013 (82.7\%) of all presentations in primary care. Nurse practitioners, as the first contact primary care providers, are expected to assess and manage these RFE. The top reason for encounter overall was ongoing care for hypertension.

Out of these 80 RFE Table 4 shows the distribution of follow up appointments for medication, examination and results. Chronic or ongoing care visits made up $8896(28.4 \%)$ of these top $80 \mathrm{RFE}$. The second largest contributor to chronic care was women's health which included family planning and pregnancy related consultations. Chronic care for non-communicable chronic diseases made up at least $4344(13.9 \%)$ of all reasons for encounter.

Out of the top 80 RFE Table 5 lists the commonest symptoms presented to primary care providers. Primary care providers need to have an approach to assessing and diagnosing patients who present with these undifferentiated complaints. Trauma and injury only compromised $92(0.3 \%)$ of all RFE despite being the second largest contributor to the burden of disease. This would imply that trauma and injury is usually seen elsewhere, presumably in emergency rooms and hospital settings.

Table 6 shows the top 20 RFE by age group and allows a comparison between the under-5s, 5-14 years and 15 years and older. 
Table 6. Top 20 RFE by age group.

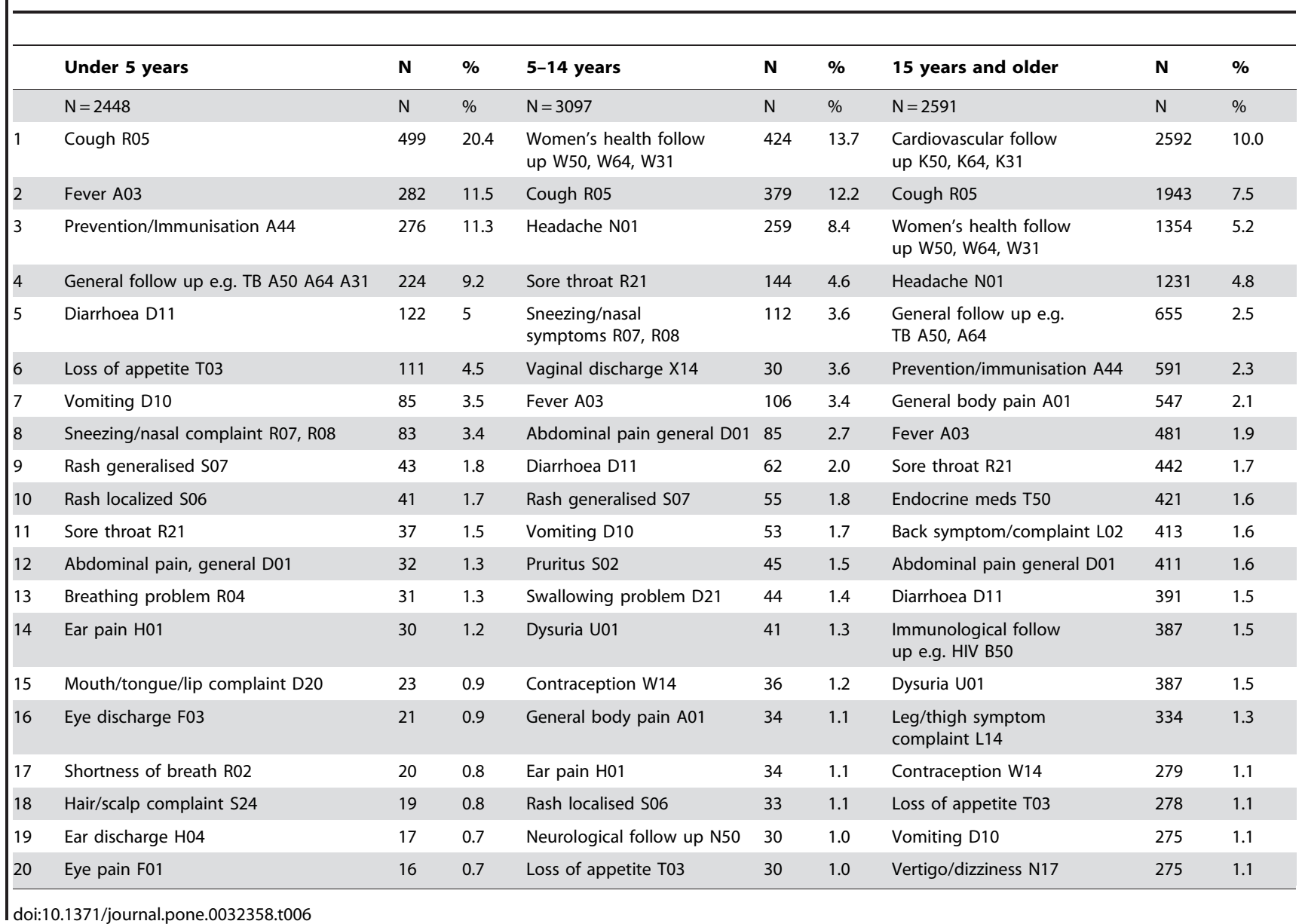

Figure 2 shows the pattern of selected RFE from the top 20 by age group. Figure 2a shows that cough peaks in the under-5s and then gradually declines, although it remains common in all age groups. Headache is a common symptom in all age groups. Dysuria is also found in all age groups with a small peak in the 2024 year old bracket. Back pain gradually increases with age and becomes relatively stable as a symptom after the age of 30-years.

Figure $2 \mathrm{~b}$ shows that fever starts at a peak in the under- $5 \mathrm{~s}$, falls rapidly over the next 5 -years and then levels out to decline more slowly over the adult years. Diarrhoea, vomiting and generalised abdominal pain or cramps follow a similar although less dramatic pattern. Generalised body pain is a constant feature in all age groups but tends to increase gradually with age.

Figure 2c shows that visits for women's health issues (family planning) peak in the 10-14 year old age group. HIV and TB peak in the 30-34 year old age group and TB also shows a peak in the under-5s. Cardiovascular (mostly hypertension) rises progressively from the teenage years to peak in the 60-64 year age group. Diabetes follows a similar pattern.

There were no major differences between the top 25 RFE between men and women apart from women's health visits for family planning, pregnancy and vaginal discharge.

The top 25 diagnoses are listed in Table 7 and represent 13065 $(53.2 \%)$ of all diagnoses found in primary care. Hypertension is the commonest diagnosis by far, especially when uncomplicated and complicated cases are combined to give $3219(13.1 \%)$ of all diagnoses. ICPC-2 does not provide codes for syndromic diagnosis of sexually transmitted infections and therefore the code for "Infectious disease, other" was used to code for sexually transmitted infection.

No psychiatric diagnoses appeared in the top 25 and the commonest diagnosis was schizophrenia (83, 0.3\%). Depression $(54,0.2 \%)$ and anxiety disorders $(19,0.1 \%)$ were less commonly diagnosed than schizophrenia. Injury and trauma are also absent from the top 25 diagnoses. There were no significant differences in the top 25 diagnoses made in men and women apart from family planning and pregnancy amongst women and COPD amongst men.

Table 8 shows the top 20 diagnoses by age group.

Figure $3 \mathrm{a}$ and $3 \mathrm{~b}$ shows the distribution of common infectious diseases with age. Tonsillitis reaches a sharp peak in the 5-9 year old age group and then gradually declines. Gastroenteritis and pneumonia starts with a peak in the under- 5 age group, then falls sharply over the next 5 -years and afterwards slowly declines. Bronchitis and lower respiratory tract infections have a fairly constant frequency in all age groups. Urinary tract infections are present in all age groups but peak in the 20-24 year category. Sexually transmitted infections peak in the 25-29 year old age group and then drop sharply. HIV/AIDS and TB peak in the 2534 year old age group.

Figure 3c shows the distribution of diagnoses for noncommunicable diseases across age groups. Hypertension climbs continuously to reach a plateau in the 60-64 year age group. Asthma peaks in the 5-9 year old age group and then again in the 50-54 year old age group. Type 2 diabetes climbs to a peak in the 60-64 year old age group. Epilepsy has a constant presence from 


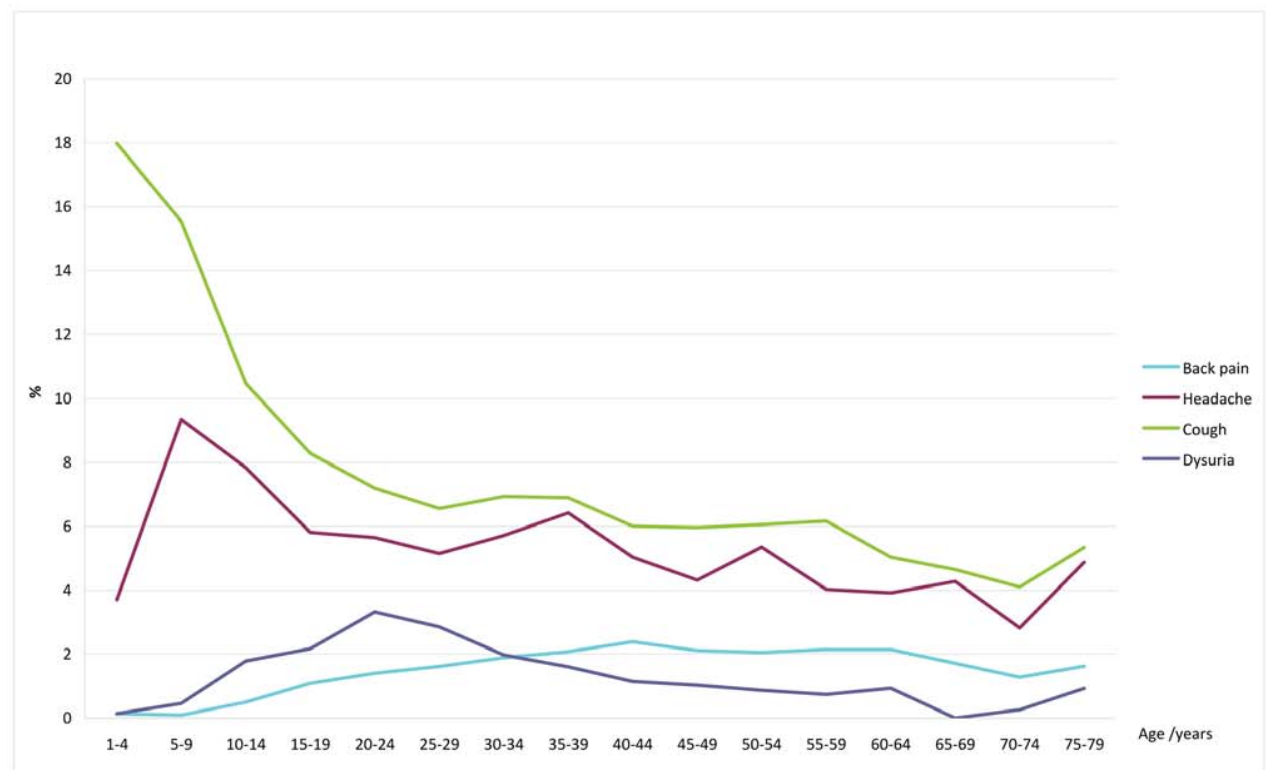

12

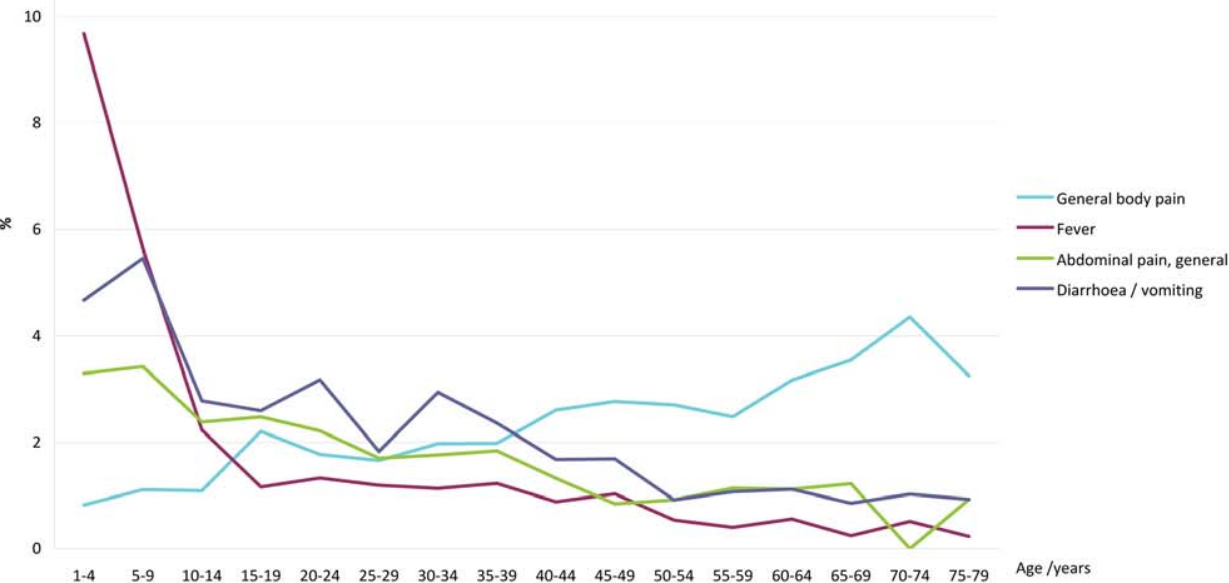

25

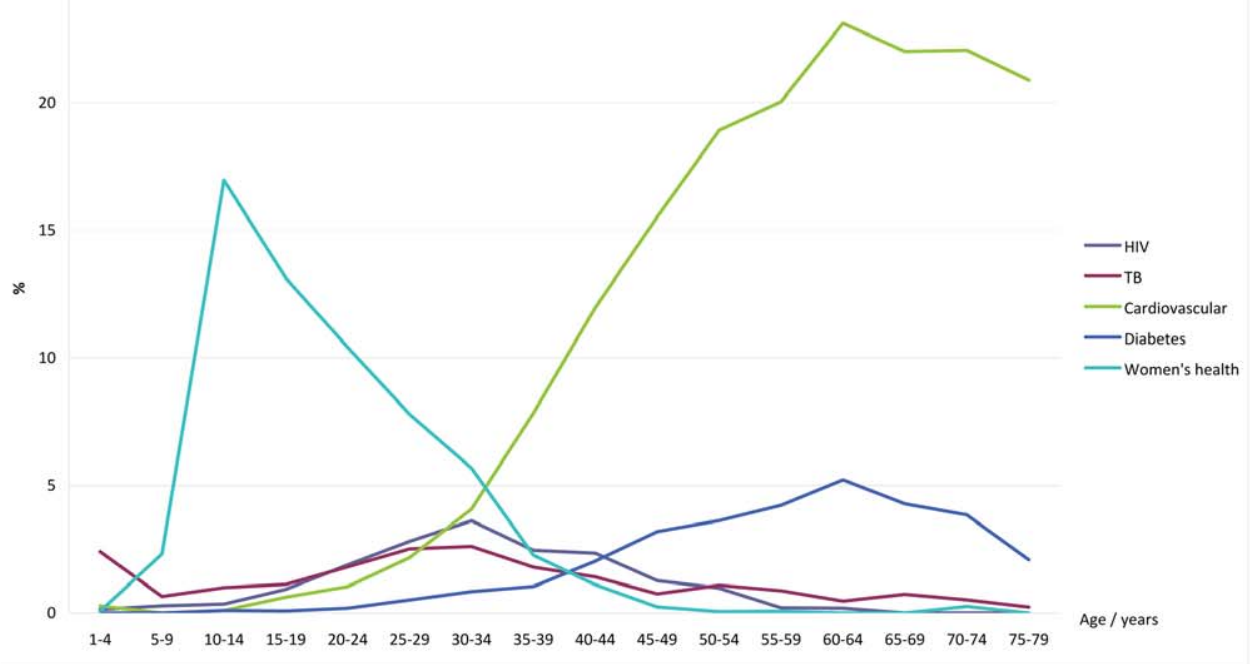

Figure 2. Patterns of selected reasons for encounter by age.

doi:10.1371/journal.pone.0032358.g002

PLoS ONE I www.plosone.org 
Table 7. Top 25 diagnoses in South African primary care $(\mathrm{N}=24561)$.

\begin{tabular}{|c|c|c|}
\hline Diagnosis & $\mathbf{n}$ & $\%$ \\
\hline Hypertension, uncomplicated (K86) & 2957 & 12.0 \\
\hline Upper respiratory tract infection (R74) & 1306 & 5.3 \\
\hline HIV/AIDS (B90) & 961 & 3.9 \\
\hline Type 2 diabetes (T90) & 946 & 3.9 \\
\hline TB (A70) & 862 & 3.6 \\
\hline Cough (R05) & 681 & 2.8 \\
\hline Osteoarthritis (L91) & 530 & 2.2 \\
\hline Gastroenteritis/diarrhoea (D73, D11) & 491 & 2.0 \\
\hline Asthma (R96) & 485 & 2.0 \\
\hline Acute tonsillitis (R76) & 454 & 1.9 \\
\hline Epilepsy (N88) & 375 & 1.5 \\
\hline Infectious disease, other (A78) & 366 & 1.5 \\
\hline Urinary tract infection (U71) & 317 & 1.3 \\
\hline Pneumonia (R81) & 306 & 1.2 \\
\hline Acute bronchitis/bronchiolitis (R78) & 263 & 1.1 \\
\hline Hypertension, complicated (K87) & 262 & 1.1 \\
\hline Acute otitis media (H71) & 233 & 0.9 \\
\hline Generalised body pain (A01) & 213 & 0.9 \\
\hline Headache (N01) & 209 & 0.9 \\
\hline Influenza (R80) & 189 & 0.8 \\
\hline Muscle pain (L18) & 183 & 0.7 \\
\hline Allergic reaction (A92) & 176 & 0.7 \\
\hline Dermatophytosis (S74) & 160 & 0.7 \\
\hline Chronic obstructive pulmonary disease (R95) & 140 & 0.6 \\
\hline
\end{tabular}

the 5-9 year old age group and reduces somewhat from 5559 years onwards.

\section{Discussion}

\section{Key findings}

The findings reflect current morbidity found in South African ambulatory primary care. The late teens/young adult age groups predominate and this reflects the age distribution within the South African population. The adolescent population had amongst the highest number of consultations, which was a surprising result as this is usually a relatively healthy group. The high number of consultations appeared due to sexual health (contraception and pregnancy), HIV and related infections (STIs, TB, pneumonia, diarrhoea). Visits for contraception peaked in the 10-14 year age group. This finding may indicate that more attention be given to the special needs of adolescents in the design of health services as current programmes focus more on young children or adults.

Women accounted for a greater percentage of consultations than in other primary care settings [13] The reasons for this are unclear, but could be due to primary care taking more responsibility for reproductive health services or the higher prevalence of HIV amongst young women.[14]

The overwhelming majority of patients were seen by nurses. Doctors saw a much smaller percentage of patients, which is consistent with their usual role of just seeing patients referred to them by the nurses. Many clinics are only visited once a week by doctors. The higher mean number of RFE and diagnoses suggests that these patients had multi-morbidity and were probably more complicated than the patients seen by nurses.

If one compares the experience of primary care with the estimated burden of disease in South Africa there are some notable differences. HIV and TB as well as child health issues (pneumonia, diarrhoea) are well represented in primary care practice as they fall within the top 25 diagnoses. However as HIV is by far the leading cause of premature mortality and morbidity and TB the third leading cause one would have expected these to be more prominent in terms of diagnoses and chronic care visits. [5] The reason for this is probably because most patients with HIV and TB are not offered ongoing primary care, but are seen in specialised clinics and separate vertical programmes. Low birth weight and birth trauma/asphyxia are not seen, but would not be expected in an ambulatory primary care setting. Interpersonal violence (assault, injuries) and road traffic accidents which make a huge contribution to the burden of disease [5] are also rarely seen and will mostly likely present to emergency rooms and hospital settings. However the very low recognition of interpersonal violence as an issue is worrying as intimate partner violence is a large component of this in women and usually presents with psychological and other symptoms. [15] Non communicable chronic diseases are more prominent in primary care than expected from the burden of disease, especially hypertension and type 2 diabetes. Hypertension alone is the leading reason to attend primary care and the most common diagnosis, even in a context in which it is estimated that only $26 \%$ of men and $51 \%$ of women people are aware of their hypertension. [16] Mental disorders and substance abuse are not recognised or diagnosed, which is a major omission, as the WHO estimates that up to $24 \%$ of consultations in primary care include a mental disorder such as depression, anxiety or alcohol abuse. [17] The South African Stress and Health Survey estimated that 16.5\% of people had a 12-month prevalence of a mental health disorder and that $26.2 \%$ of these were severe disorders. Depression, anxiety and alcohol use disorders were the commonest disorders found. [18] Problems such as deafness and cataracts, which appear in the top 20 contributors to the burden of disease, may also be poorly recognised.

The majority of patients were seen by nurses and not all were trained clinical nurse practitioners. Even clinical nurse practitioners only receive an additional 1 -year of training to cope with the range of problems seen in primary care. The survey highlights the need to ensure that nurses are trained and competent to handle the common problems and raises the question of whether more consultations should be with doctors. The accuracy of their diagnoses cannot be determined from this data. However if nurses are expected to manage the range of diseases found in the survey they should also be enabled to treat them appropriately. For example in many provinces professional nurses are only allowed to prescribe hydrochlorothiazide for hypertension. The current revitalisation of primary care has to balance increasing availability of primary care services through nurse-led primary care teams with improving the acceptability and quality of those services. Family physicians and doctors may need to play a more active role in terms of mentoring and support.

A number of symptoms were used to provide diagnoses: cough, diarrhoea, generalised body pain, headache and muscle pain. In some cases this may represent an inability or unwillingness to make a more specific assessment or diagnosis. For example headache was rarely diagnosed in a more specific way such as tension headache or migraine. Generalised body pain is often a difficult presentation to make sense of or assess. 
Table 8. Top 20 diagnoses by age group.

\begin{tabular}{|c|c|c|c|c|c|c|c|c|c|}
\hline & Under 5 year & $\mathbf{N}$ & $\%$ & $5-14$ years & $\mathbf{N}$ & $\%$ & 15 years and older & $\mathbf{N}$ & $\%$ \\
\hline & $N=1697$ & $\mathrm{~N}$ & $\%$ & $N=2242$ & $\mathrm{~N}$ & $\%$ & $N=20622$ & $\mathrm{~N}$ & $\%$ \\
\hline 1 & URTI R74 & 255 & 15.0 & Contraception W14 & 303 & 13.5 & Hypertension K86 & 2954 & 14.3 \\
\hline 2 & Health maintenance/prevention A98 & 169 & 10.0 & URTI R74 & 202 & 9.0 & Diabetes T90 & 942 & 4.6 \\
\hline 3 & Cough R05 & 121 & 7.1 & Pregnancy W78 & 167 & 7.3 & HIV B90 & 912 & 4.4 \\
\hline 4 & Pneumonia R81 & 113 & 6.7 & Cough R05 & 125 & 5.6 & Contraception W14 & 878 & 4.3 \\
\hline 5 & Immunisation A44 & 103 & 6.1 & Acute tonsillitis R76 & 110 & 4.9 & URTI R74 & 849 & 4.1 \\
\hline 6 & Diarrhoea D11 & 65 & 3.8 & TB A70 & 57 & 2.5 & TB A70 & 743 & 3.6 \\
\hline 7 & TB A70 & 63 & 3.7 & Headache N01 & 46 & 2.1 & Pregnancy W78 & 609 & 3.0 \\
\hline 8 & Acute otitis media $\mathrm{H} 71$ & 44 & 2.6 & Epilepsy N88 & 41 & 1.8 & Osteoarthritis L91 & 527 & 2.6 \\
\hline 9 & Acute tonsillitis R76 & 41 & 2.4 & HIV B90 & 37 & 1.7 & Asthma R96 & 446 & 2.2 \\
\hline 10 & Fever $\mathrm{A} 03$ & 40 & 2.4 & Asthma R96 & 31 & 1.4 & Cough R05 & 435 & 2.1 \\
\hline 11 & Gastroenteritis D73 & 35 & 2.1 & $\begin{array}{l}\text { Infectious disease, } \\
\text { other A78 }\end{array}$ & 30 & 1.3 & $\begin{array}{l}\text { Health maintenance/prevention } \\
\text { A98 }\end{array}$ & 354 & 1.7 \\
\hline 12 & Dermatophytosis S74 & 30 & 1.8 & Pneumonia R81 & 30 & 1.3 & Infectious disease, other A78 & 330 & 1.6 \\
\hline 13 & Impetigo S84 & 26 & 1.5 & Influenza R80 & 28 & 1.2 & Epilepsy N88 & 330 & 1.6 \\
\hline 14 & Vitamin/nutrition deficiency T91 & 25 & 1.5 & Diarrhoea D11 & 27 & 1.2 & Acute tonsillitis R76 & 303 & 1.5 \\
\hline 15 & Mouth/tongue/lip disease D83 & 23 & 1.4 & $\begin{array}{l}\text { Allergy/allergic } \\
\text { reaction A92 }\end{array}$ & 26 & 1.2 & UTI U71 & 289 & 1.4 \\
\hline 16 & No disease $A 97$ & 22 & 1.3 & $\begin{array}{l}\text { Abdominal pain } \\
\text { general D01 }\end{array}$ & 26 & 1.2 & Hypertension, complicated K87 & 262 & 1.3 \\
\hline 17 & Allergy/allergic reaction A92 & 21 & 1.2 & UTI U71 & 26 & 1.2 & Acute bronchitis R78 & 225 & 1.1 \\
\hline 18 & Worms/other parasites D96 & 19 & 1.1 & Gastroenteritis D73 & 25 & 1.1 & Prevention/Immunisation A44 & 219 & 1.1 \\
\hline 19 & Influenza R80 & 19 & 1.1 & Acute otitis media $\mathrm{H} 71$ & 123 & 1.0 & General body pain A01 & 196 & 1.0 \\
\hline 20 & Conjunctivitis infectious F70 & 17 & 1.0 & Acute bronchitis R78 & 22 & 1.0 & Gastroenteritis D73 & 195 & 0.9 \\
\hline
\end{tabular}

doi:10.1371/journal.pone.0032358.t008

\section{Comparison to the literature}

Compared to a similar survey performed in 2001-2 in the Eastern Cape of South Africa non-communicable chronic diseases and HIV/AIDS have both increased significantly amongst the reasons for attendance and diagnoses over the last 10-years. [7] For example cardiovascular reasons for attendance increased from fourteenth in 2001 to the third most common in 2010, while blood and immune reasons (mainly HIV) increased from seventeenth in 2001 to eleventh in 2010. It is surprising that HIV was not more prominent in both surveys. In 2001 the researchers believe this may have been due to a reluctance to record or diagnose it at that time while in 2010 it is most likely due to the treatment of HIV in separate vertical programmes.

When the reasons for encounter by ICPC chapter are compared with other countries such as the Netherlands, Poland, Japan and USA there are a number of similarities and differences. [13] Respiratory, digestive, skin, endocrine, female and male genital, urological, eye, ear and social reasons for encounter are similar in frequency. Psychological reasons for encounter are much higher in Netherlands and USA where they are within the top five chapters. Musculoskeletal complaints are also slightly higher in these other countries. Pregnancy and family planning related reasons for encounter are much higher in South Africa showing the important role that primary care plays in this area. Blood/immune (including HIV), general unspecified and neurological reasons are also higher in South Africa. Cardiovascular is similar across all countries except for the USA where it is much less a feature of primary care. There are many possible reasons for these differences including the health systems, cultural differences and the burden of disease.
When the top 52 symptoms/complaints from the Netherlands, Poland, Japan and USA are compared to the top 56 South African the majority are the same. [13] However in these other countries psychological complaints are found (feeling depressed, anxious, sleep disturbance) as well as complaints often associated with the elderly (vision problems, hearing complaints, blocked ears), which do not appear on the South African list. In contrast a number of complaints appear on the South African list that probably reflect the burden of disease from HIV/AIDS and TB (weight loss, sweating, loss of appetite, abnormal sputum, respiratory pain, dysphagia) and STIs (genital/pelvic pain, vaginal and urethral discharge, vaginal symptoms), which do not appear in these other countries. In addition infective complaints associated with the eye and ear (eye pain and discharge, red eyes, ear discharge), trauma/ injury as well as absent or scanty menses are also listed. These may reflect the different burden of disease and more prominent role of primary care in pregnancy and family planning. The complaint of generalised/multiple body pain is also a particular feature of South African primary care and may reflect local cultural expressions of illness.

When the top 25 diagnoses are compared between these same countries and South Africa there is much less similarity. [13] In South Africa a number of diagnoses are found which do not appear in the top 25 from these other countries: HIV, TB, STIs, pneumonia, gastroenteritis, urinary tract infection, epilepsy and chronic obstructive airways disease. In these other countries the following diagnoses are found which do not feature on the South African list: sinusitis, osteoporosis, back pain, neck symptom/ complaint, gastro-oesophageal reflux, peptic ulceration, gastritis, 
10

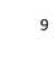

8

7

6

×
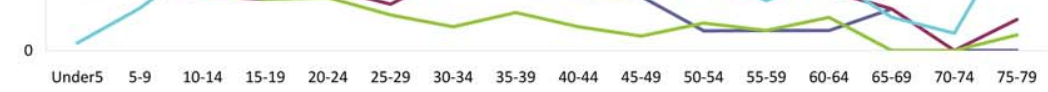

12

10

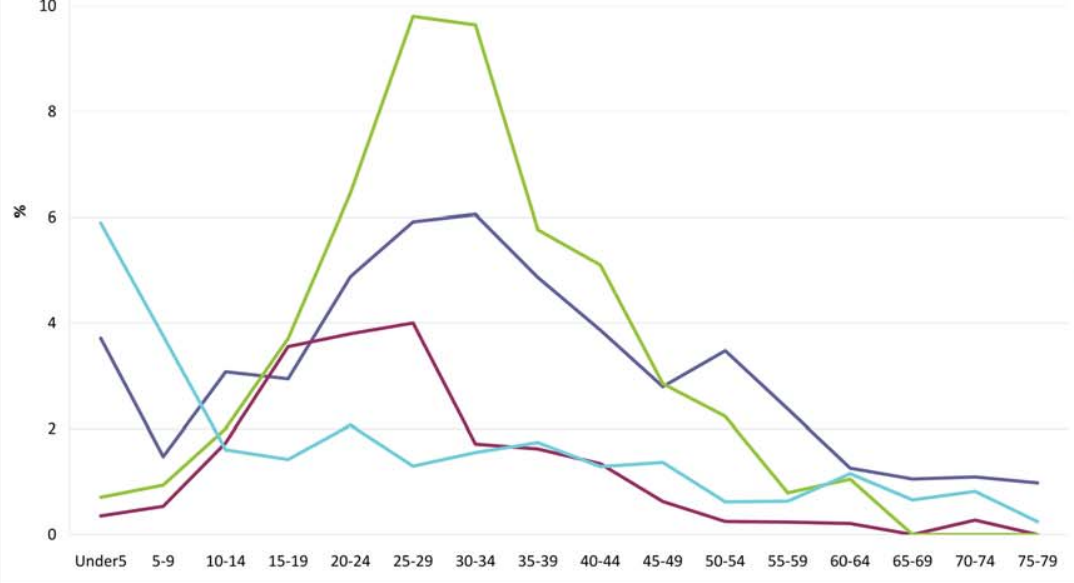

40
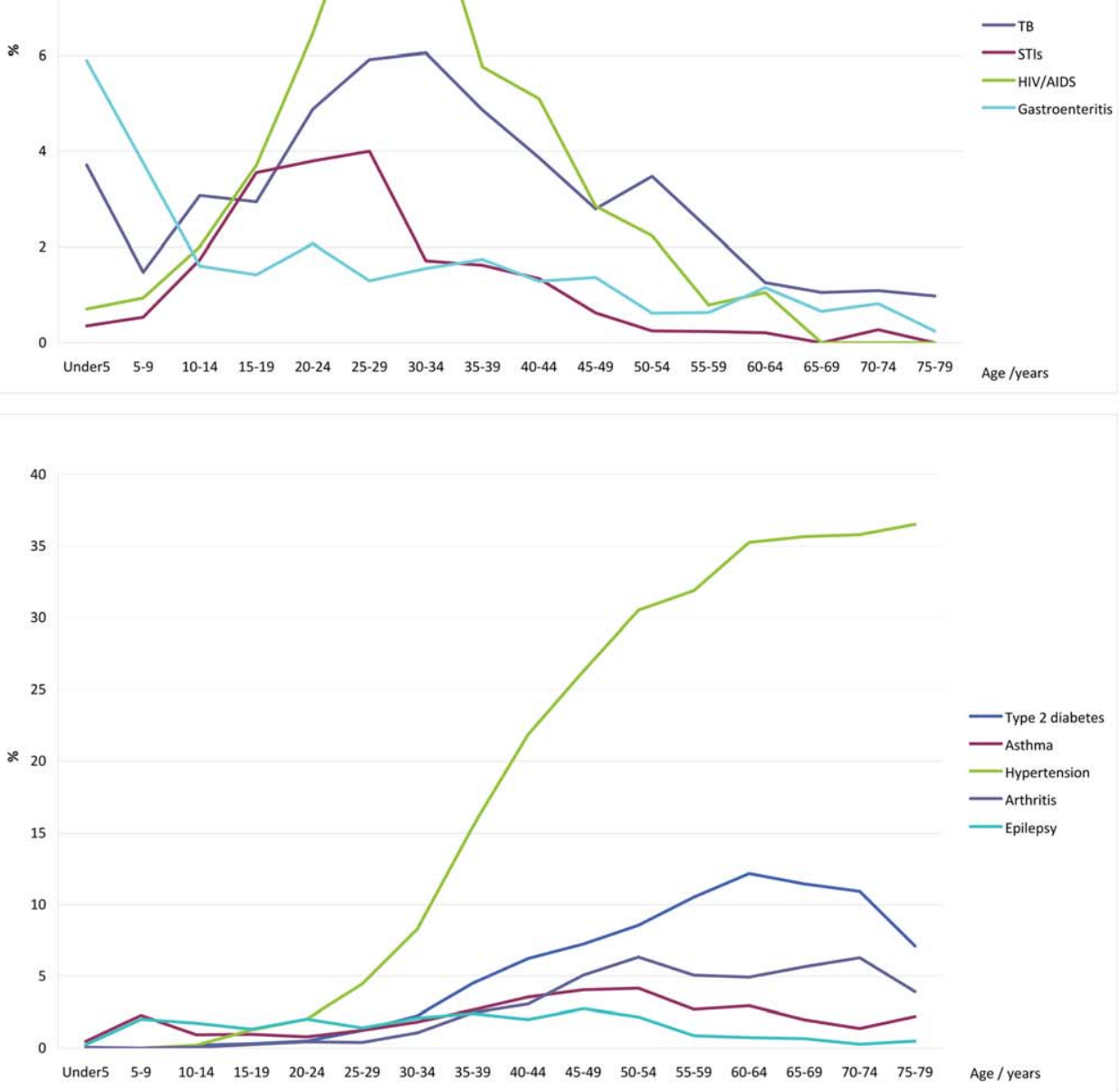

Age /years

Figure 3. Patterns of selected diagnoses by age.

doi:10.1371/journal.pone.0032358.g003

PLOS ONE I www.plosone.org 
dermatitis, sleeping problem, depression, stroke, ischaemic heart disease, lipid metabolism disorder, laceration. This demonstrates quite substantial differences in the burden of disease encountered in South African primary care and the relatively high prevalence of infective and communicable diseases in South Africa compared to these countries. It again emphasises that mental health problems are under diagnosed. The prominence of COPD may be related to chronic lung disease from TB in addition to tobacco smoking.

\section{Strengths and limitations}

The survey was not performed in all provinces of South Africa and it is possible that a different pattern could be found elsewhere. In addition districts and sub-districts were purposefully and not randomly selected within provinces, which could influence the results, should other sub-districts be significantly different, although this is not considered likely. Data from provinces was combined without stratification for differences in population size between provinces. This is the largest such survey performed in South Africa to date. Although the full sample size was not obtained the total number of consultations was sufficient to provide information on the prevalence of the commonest RFE and diagnoses. The sample size from the Northern Cape was a lot less than expected and was mainly due to a shortage of anticipated staff members to participate in the survey at each facility. The top RFE and diagnoses from the Northern Cape did not differ substantially from the rest of the survey and there is no reason to think that a larger sample would have changed the overall results. Errors in coding were often due to relatively minor differences, such as between R07 (sneezing/nasal congestion) and R08 (nasal symptoms/other) or to omissions such as when a recorded RFE was not coded. The article only reports on the commonest RFE and diagnoses where the error rate is likely to have less impact on the ranking of items. The accuracy of diagnoses cannot be determined.

\section{Implications and recommendations}

The profile of primary care will inform the curriculum for training of primary care nurses, medical students and family physicians as this represents the presentations to which primary care providers must have an evidence based and effective approach. The profile should also influence the development of tools and content of educational resources, for example the recent expansion of the Practical Approach to Lung Health and HIV/ AIDS guideline to include non-communicable diseases, mental health and antenatal care. The results highlight the need for more attention to psychological and social aspects of care in the training of primary care providers as well as the need for skills in ongoing and chronic care. The profile will also inform the assessment of these providers, for example in the exam offered by the College of Family Physicians.

\section{References}

1. World Health Organisation (2008) World Health Report - Primary Health Care: Now more than ever. Geneva: World Health Organisation.

2. Rispel L, Moorman J, Chersich M, Goudge J, Nxumalo N, etal (2010) Revitalising primary health care in South Africa: Review of primary health care package, norms and standard. Johannesburg: Centre for Health Policy, University of the Witwatersrand.

3. Hugo J, Allan L (2008) Doctors for tomorrow: family medicine in South Africa. Grahamstown: National Inquiry Services Centre.

4. Harris M, Haines A (2010) Brazil's family health programme BMJ; 341: c4945.

5. Bradshaw D, Norman R, Schneider M (2007) A clarion call for action based on refined DALY estimates for South Africa. S Afr Med J 976: 438-440.
The study provides useful feedback to district managers on the current focus of ambulatory primary care and can enable reflection on the direction of in-service training, allocation of resources and future organisation of care. It also reflects the vertical nature of HIV services, which exacerbates the problem of fragmented care for those surviving many years due to antiretroviral treatment, and who find themselves at increased risk of developing non-communicable disease and mental health problems. Already South Africa's Ministry of Health is exploring models whereby all chronic conditions, whether non-communicable, infectious or psychological could be integrated into a single chronic care service. Further analysis of the data set will be possible to explore what diagnoses primary care providers make from these presentations and to calculate the likelihood ratios of different conditions. For example how do primary care providers make sense of generalised body pain? It will also be possible to explore what presentations are commonly associated with specific diagnoses such as HIV/AIDS or depression and what diagnoses are commonly associated with each other.

\section{Conclusion}

The survey presents a profile of morbidity in South African primary care and identifies the commonest reasons for encounter and diagnoses made. Ambulatory primary care is dominated by non-communicable chronic diseases such as hypertension and diabetes. HIV/AIDS and TB are present, but not to the extent predicted by the burden of disease, this is most likely because they are treated in separate vertical programmes. Pneumonia and gastroenteritis are commonly seen especially in children. Women's health issues such as family planning and pregnancy related visits are also common. Injuries are not as common as expected from the burden of disease and this is most likely because they present to emergency units. However it is also likely that intimate partner violence is unrecognised in primary care and providers appears to be failing to recognise and treat mental health problems such as depression and anxiety disorders. The results should guide the future training and assessment of primary care providers.

\section{Acknowledgments}

Prof Carl Lombard, Head of Biostatistics at the Medical Research Council, South Africa, for assisting with the sampling design and reading the manuscript. Mr Justin Harvey, Statistician, Centre for Statistical Consultation, Stellenbosch University, for analysing the data. Dr Ruth Cornick assisted with the provision of vouchers and initial preparation of the study.

\section{Author Contributions}

Conceived and designed the experiments: BM LF. Performed the experiments: SM JK RO OI OA WY. Analyzed the data: BM LF SM JK RO OI OA WY. Contributed reagents/materials/analysis tools: BM. Wrote the paper: BM LF. Approved final manuscript: SM JK RO OI OA WY.

6. De Villiers P, Du Plessis J, Saban J, De Villiers M, Reid A, et al. (1994) What is happening in Cape Town Family Medicine/Primary Care Practice? S Afr Fam Pract 15: 363-372.

7. Brueton V, Yogeswaran P, Chandia J, Mfenyana K, Modell B, et al. (2010) Primary care morbidity in Eastern Cape province. S Afr Med J 1005: 309-312.

8. Silbert M (1970) The Cape Morbidity Survey and its significance in the training for general practice. S Afr Med J 448: 28.

9. Bloom B, Bourne D, Sayed A, Klopper J (1988) Morbidity patterns from general practice in Cape Town- a pilot study. S Afr Med J 73: 166-168.

10. Department of Health (2011) National Health Insurance in South Africa: Policy Paper. Government Gazette 554: 34523. 
11. Couper I (2006) The rural doctor in Mash B editor. Handbook of Family Medicine. Cape Town: Oxford University Press; 298-315.

12. WONCA International Classification Committee (1998) International Classification of Primary Care (ICPC-2). Oxford: Oxford University Press.

13. Okkes IM, Polderman GO, Fryer GE, Yamada T, Bujak M, et al. (2002) The role of family practice in different health care systems. A comparison of reasons for encounter, diagnoses, and interventions in primary care populations in the Netherlands, Japan, Poland, and the United States. J Fam Pract 51: 72.

14. Ross D, Dick B, Ferguson J (2006) Preventing HIV/AIDS in young people: a systematic review of the evidence from developing countries. Geneva: WHO Technical Report Series. 938 p.
15. Joyner K (2010) Aspects of Forensic Medicine - An Introduction for Healthcare Professionals. Cape Town: Juta Health.

16. Rayner B (2010) Hypertension: Detection and Management in South Africa. Nephron Clinical Practice 1164: c269-c273.

17. Ustun TB, Sartorius N (1995) Mental Illness in General Health Care: An International Study. Chichester: Wiley \& Sons.

18. Williams DR, Herman A, Stein DJ, Heeringa SG, Jackson PB, et al. (2008) Twelve-month mental disorders in South Africa: prevalence, service use and demographic correlates in the population-based South African Stress and Health Study. Psychol Med 382: 211-20. 